\begin{tabular}{|c|l|}
\hline Title & Photodisintegration cross sections for resonant states and virtual states \\
\hline Author(s) & Odsuren, Myagmarjav; Kikuchi, Y uma; Myo, Takay uki; Masui, Hiroshi; Katō , Kiyoshi \\
\hline Citation & $\begin{array}{l}\text { Physical Review C, 99(3), 034312 } \\
\text { https://doi.org/10.1103/PhysRevC.99.034312 }\end{array}$ \\
\hline Issue Date & 2019-03-13 \\
\hline Doc URL & http://hdl.handle.net/2115/73483 \\
\hline Rights & @2019American Physical Society \\
\hline Type & article \\
\hline File Information & PhysRevC.99-3_034312.pdf \\
\hline
\end{tabular}

Instructions for use 


\title{
Photodisintegration cross sections for resonant states and virtual states
}

\author{
Myagmarjav Odsuren, ${ }^{1, *}$ Yuma Kikuchi, ${ }^{2, \dagger}$ Takayuki Myo, ${ }^{3,4, \$}$ Hiroshi Masui, ${ }^{5, \S}$ and Kiyoshi Katōo ${ }^{6, \|}$ \\ ${ }^{1}$ School of Engineering and Applied Sciences and Nuclear Research Center, National University of Mongolia, \\ Ulaanbaatar 210646, Mongolia \\ ${ }^{2}$ Tokuyama College, National Institute of Technology, Yamaguchi 745-8585, Japan \\ ${ }^{3}$ Faculty of Engineering, General Education, Osaka Institute of Technology, Osaka 535-8585, Japan \\ ${ }^{4}$ Research Center for Nuclear Physics (RCNP), Osaka University, Ibaraki 567-0047, Japan \\ ${ }^{5}$ Information Processing Center, Kitami Institute of Technology, Kitami 090-8507, Japan \\ ${ }^{6}$ Nuclear Reaction Data Centre, Faculty of Science, Hokkaido University, Sapporo 060-0810, Japan
}

(Received 4 November 2018; published 13 March 2019)

\begin{abstract}
The $s$-wave photodisintegration cross sections observed just above the neutron breakup threshold energy are calculated for resonant and virtual states by using a complex-scaled two-body model, which simulates the ${ }^{9} \mathrm{Be}$ nucleus with a ${ }^{8} \mathrm{Be}+n s$-wave channel. We investigate the differences between behaviors of the resonance and virtual states in the photodisintegration cross sections near threshold energy in detail. It is shown that, in the present two-body model, resonant states very near the threshold energy produce a peak structure in the photodisintegration cross section that is similar that of the virtual state.
\end{abstract}

DOI: 10.1103/PhysRevC.99.034312

\section{INTRODUCTION}

Nuclear states observed around threshold energies provide us with interesting problems associated with the nuclear cluster structure [1,2]. Most of them are also interesting astrophysically from the viewpoint of nucleosynthesis. The Hoyle state [3] of the excited $0^{+}$state in ${ }^{12} \mathrm{C}$ is one of the typical examples in light nuclei. The first excited $J^{\pi}=1 / 2^{+}$state in ${ }^{9} \mathrm{Be}[4]$, which is an $\alpha+\alpha+n$ Borromean nucleus, offers us a similar problem. This state of ${ }^{9} \mathrm{Be}$ has been observed as a sharp peak above the ${ }^{8} \mathrm{Be}+n$ threshold energy in the photodisintegration cross section of $\gamma+{ }^{9} \mathrm{Be} \rightarrow \alpha+\alpha+n$. The strength of the peak has a strong influence on the reaction rate of the ${ }^{9} \mathrm{Be}$ synthesis. From such an astrophysical interest, new experiments and their analyses were performed recently [5-7].

From theoretical studies, nuclear structure of this state of ${ }^{9} \mathrm{Be}$ has been discussed from various points of view: ${ }^{8} \mathrm{Be}+n$ cluster model [8,9], $\alpha+\alpha+n$ three-body model [10,11], molecular orbit model of a valence neutron around two $\alpha$ clusters [12-14], and so on. There is a long-standing controversy whether the $1 / 2^{+}$state is a resonant or a virtual state [10,15-17]. Recently, we performed the calculations using an $\alpha+\alpha+n$ three-body model $[18,19]$ together with the complex scaling method (CSM) [20], which well reproduces the recently observed photodisintegration cross section $[5,6]$. The results indicate that the $1 / 2^{+}$state shows the $s$-wave virtual-state character of ${ }^{8} \mathrm{Be}+n$, which plays an important role in formation of the peak structure above the ${ }^{8} \mathrm{Be}+n$ threshold in the cross section $[18,19]$.
However, the virtual state cannot be directly obtained as an isolated pole solution in the CSM [21,22], because the scaling angle in the CSM cannot cover the position of the virtual state pole on the negative imaginary axis of the complex momentum plane [22]. In our previous work [23], we proposed a new approach for the CSM to describe the virtual state. We showed that the CSM is also useful to distinguish a virtual state pole from the continuum using the continuum level density (CLD), the scattering phase shift, and the scattering length [23]. In the next step, we investigate the differences between behaviors of the virtual state and resonance in the photodisintegration cross sections.

For this purpose, we apply the CSM to the two-body model, ${ }^{8} \mathrm{Be}+n$, used in our previous calculation for ${ }^{9} \mathrm{Be}$ [23], and further we discuss how the two-body unbound state contributes to the photodisintegration cross section of ${ }^{9} \mathrm{Be}$. This potential model well describes the $p$ - and $s$-wave neutron motions around the ${ }^{8} \mathrm{Be}$ quasicluster, which correspond to the ground and excited states of ${ }^{9} \mathrm{Be}$, respectively. We investigate the $E 1$ transition strength causing the photodisintegration by changing the potential strength in the two-body system. We precisely discuss the origin of the peak of the $E 1$ strength near the threshold in relation to the virtual and resonant states of the ${ }^{8} \mathrm{Be}+n$ system.

In the next section (II), we explain our model and method briefly. In Sec. III, results of the E1 transition strength and the photodisintegration cross sections are presented and discussed. Finally, in Sec. IV, we give a conclusion.

\footnotetext{
*odsuren@seas.num.edu.mn

${ }^{\dagger}$ kikuchi@tokuyama.ac.jp

†takayuki.myo@oit.ac.jp

§hgmasui@mail.kitami-it.ac.jp

"kato-iku@gd6.so-net.ne.jp
}

\section{MODEL AND METHOD}

\section{A. A simple model in the complex scaling method}

To understand the origin of a low-energy peak in the photodisintegration cross section just above the breakup threshold 
energy in ${ }^{9} \mathrm{Be}$, we investigate a simple schematic two-body model which simulates the ${ }^{8} \mathrm{Be}+n$ system. In this schematic two-body model, as shown in Ref. [23], both clusters $\left({ }^{8} \mathrm{Be}\right.$ and $n$ ) are assumed to be structureless and spinless, and the relative motion between those clusters is described by the following Schrödinger equation:

$$
H \Psi_{J^{\pi}}^{v}=E_{v} \Psi_{J^{\pi}}^{v}
$$

where $J^{\pi}$ is the spin and parity, and $v$ is the state index. The Hamiltonian $H$ consists of the relative kinetic energy $T=-\frac{\hbar^{2}}{2 \mu} \nabla^{2}$, where $\mu$ is the reduced mass, and potential $V(r)$ for relative motion:

$$
H=-\frac{\hbar^{2}}{2 \mu} \nabla^{2}+V(r)
$$

Here, we assume the simple two-body potential

$$
V(r)=-V_{1} \exp \left(-a r^{2}\right)
$$

and we put $\frac{\hbar^{2}}{2 \mu}=1\left(\mathrm{MeV} \mathrm{fm}^{2}\right), V_{1}=-1.42 \mathrm{MeV}$, and $a=$ $0.16 \mathrm{fm}^{-2}$ so that the system has two bound states: one is the $s$ wave and the other is the $p$ wave. In order to simulate the ${ }^{8} \mathrm{Be}+n$ system, we regard the first $s$-wave bound state as the Pauli-forbidden state, and the $p$-wave bound state is assigned to the ground state of ${ }^{9} \mathrm{Be}$.

The eigenvalue problem of Eq. (1) is solved under a boundary condition of asymptotic outgoing waves for unbound states. We employ the complex scaling method (CSM) [20] which enables us to obtain the outgoing unbound states in the $L^{2}$-function basis set together with bound states $[21,22]$. In the CSM, the following complex-dilation transformation is applied to the relative coordinate $\vec{r}$ and momentum $\vec{k}$ :

$$
\vec{r} \rightarrow \vec{r} e^{i \theta}, \quad \vec{k} \rightarrow \vec{k} e^{-i \theta},
$$

where $\theta$ is a scaling angle and $0<\theta<\theta_{\max }$. The maximum value $\theta_{\max }$ is determined to keep analyticity of the potential. For example, $\theta_{\max }=\pi / 4$ for a Gaussian potential. This transformation makes every branch cut rotated by $-2 \theta$ on the complex energy plane. Applying this transformation [Eq. (4)] to Eq. (1), we can rewrite the complex-scaled Schrödinger equation as

$$
H^{\theta} \Psi_{J^{\pi}}^{\nu}(\theta)=E_{\nu}^{\theta} \Psi_{J^{\pi}}^{\nu}(\theta) .
$$

The complex-scaled Hamiltonian $H^{\theta}$ and wave function $\Psi_{J^{\pi}}^{\nu}(\theta)$ are defined as $U(\theta) H U(\theta)^{-1}$ and $U(\theta) \Psi_{J^{\pi}}^{\nu}$, respectively; see Refs. [21,22] for details. As $L^{2}$-basis functions, we employ Gaussian functions [24], and then the radial wave function is expressed as

$$
\Psi_{J^{\pi}}^{v}(\theta)=\sum_{n=1}^{N} c_{n}^{J^{\pi} v}(\theta) \phi_{n}(r),
$$

where $\left\{\phi_{n}(r)\right\}$ is the Gaussian basis function set. The expansion coefficients $c_{n}^{J^{\pi} v}$ and the complex energy eigenvalues $E_{v}^{\theta}$ are obtained by solving the complex-scaled eigenvalue problem given in Eq. (5). The complex energies of resonant states are obtained as $E_{r}=E_{r}^{\mathrm{res}}-i \Gamma_{r} / 2$, when $\tan ^{-1}\left(\Gamma_{r} / 2 E_{r}^{\mathrm{res}}\right)<$ $2 \theta$.

\section{B. Transition strength and cross section}

Using the energy eigenvalues and eigenstates of the complex-scaled Hamiltonian $H^{\theta}$, we define the complexscaled Green's function $\mathcal{G}^{\theta}\left(E ; \mathbf{r}, \mathbf{r}^{\prime}\right)$ as

$$
\mathcal{G}^{\theta}\left(E ; \mathbf{r}, \mathbf{r}^{\prime}\right)=\left\langle\mathbf{r}\left|\frac{1}{E-H^{\theta}}\right| \mathbf{r}^{\prime}\right\rangle=\sum_{\nu=1}^{N} \frac{\Psi_{J^{\pi}}^{v}(\theta) \tilde{\Psi}_{J^{\pi}}^{v}(\theta)}{E-E_{\nu}^{\theta}} .
$$

In the derivation of the right-hand side of Eq. (7), we use the extended completeness relation [21,22]. Using the complexscaled Green's function, we calculate the photodisintegration cross section.

The cross section for the electric-dipole $(E 1)$ transitions $\sigma_{E 1}^{\gamma}$ is expressed as:

$$
\sigma_{E 1}^{\gamma}\left(E_{\gamma}\right)=\frac{16 \pi^{3}}{9}\left(\frac{E_{\gamma}}{\hbar c}\right) \frac{d B\left(E 1, E_{\gamma}\right)}{d E_{\gamma}},
$$

where $E_{\gamma}$ is the incident photon energy and $B(E 1)$ is an $E 1$ transition strength. Using the CSM and the complex-scaled Green's function in Eq. (7), the $E 1$ transition strength is calculated as

$$
\begin{aligned}
& \frac{d B\left(E 1, E_{\gamma}\right)}{d E_{\gamma}} \\
& =-\frac{1}{\pi} \frac{1}{2 J_{\mathrm{gs}}+1} \operatorname{Im}\left[\sum_{v}\left\langle\tilde{\Psi}^{\mathrm{gs}}||\left(\hat{O}^{\theta}\right)^{\dagger}(E 1)|| \Psi_{J^{\pi}}^{v}(\theta)\right\rangle\right. \\
& \left.\quad \times \frac{1}{E-E_{v}^{\theta}}\left\langle\tilde{\Psi}_{J^{\pi}}^{v}(\theta)|| \hat{O}^{\theta}(E 1)|| \Psi^{\mathrm{gs}}\right\rangle\right],
\end{aligned}
$$

where $\Psi_{\mathrm{gs}}$ is the ground state wave function with spin $J_{\mathrm{gs}}$, and $\hat{O}(E 1)$ is an $E 1$ transition operator. The energy $E$ is related to $E_{\gamma}$ as $E=E_{\gamma}-E_{\mathrm{gs}}$, where $E_{g s}$ is the binding energy of the ground state measured from the threshold.

We can write Eq. (9) as

$$
\frac{d B\left(E 1, E_{\gamma}\right)}{d E_{\gamma}}=\frac{1}{2 J_{\mathrm{gs}}+1} \operatorname{Im} \sum_{\nu}\left\{M_{\nu}^{2} \tilde{\rho}_{\nu}(E)\right\},
$$

where matrix element $M_{v}^{2}$ and level density $\tilde{\rho}(E)$ are defined as

$$
M_{\nu}^{2}=\left\langle\tilde{\Psi}^{\mathrm{gs}}||\left(\hat{O}^{\theta}\right)^{\dagger}(E 1)|| \Psi_{J^{\pi}}^{v}(\theta)\right\rangle\left\langle\tilde{\Psi}_{J^{\pi}}^{v}(\theta)|| \hat{O}^{\theta}(E 1)|| \Psi^{\mathrm{gs}}\right\rangle
$$

and

$$
\tilde{\rho}_{\nu}(E)=-\frac{1}{\pi} \frac{1}{E-E_{v}^{\theta}},
$$

respectively. It is noted that $M_{v}^{2}$ and $\tilde{\rho}_{v}$ are complex numbers, and do not directly correspond to physical quantities.

\section{RESULTS AND DISCUSSIONS}

\section{A. The shape of the cross section calculated just above threshold energy}

In the CSM, resonances are obtained as solutions with complex eigenvalues in a wedge region clipped by the positive energy line and the $2 \theta$ line in the fourth quadrant of the complex energy plane. However, because of the restriction 


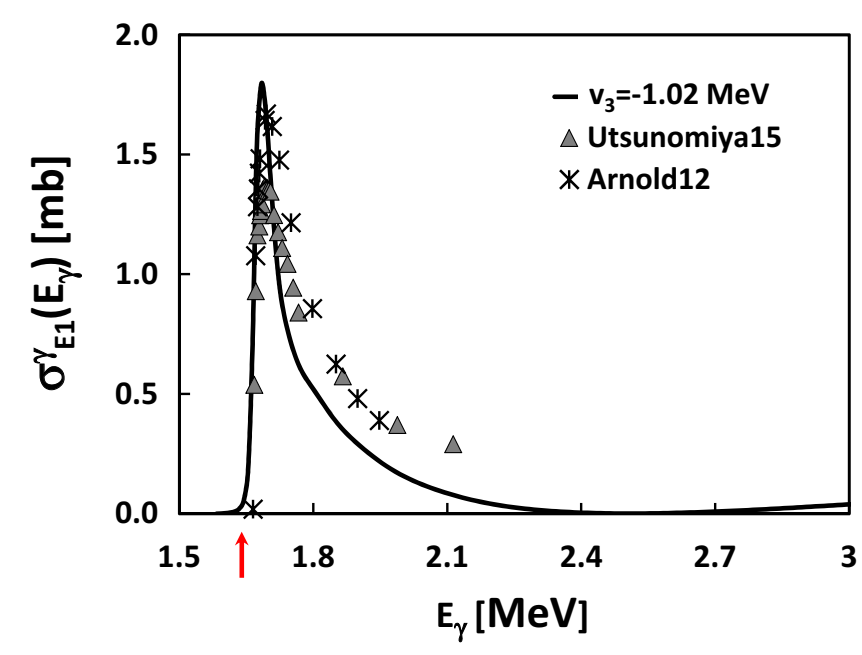

FIG. 1. The calculated photodisintegration cross section of the ${ }^{9} \mathrm{Be}\left(1 / 2^{+}\right)$, taken from Ref. [18], in comparison with experimental data $[5,6]$. The black solid line represents the cross section calculated by using the complex scaled $\alpha+\alpha+n$ model with an attractive three-body potential $v_{3}=-1.02 \mathrm{MeV}$. The arrow indicates the threshold energy of the ${ }^{8} \mathrm{Be}\left(0^{+}\right)+n$ channel.

to the scaling angle $\theta$ as $0<\theta<\theta_{\max }=\pi / 4$ coming from the analyticity of Gaussian potential, it is not possible to solve virtual state solutions, which are defined as the poles on the negative energy axis of the second Riemann sheet in the complex energy planes. The virtual-state components are considered to be buried in the continuum solutions obtained on the $2 \theta$ line [23].

In Ref. [18], we studied the $1 / 2^{+}$state of ${ }^{9} \mathrm{Be}$ and the $E 1$ photodisintegration cross section, applying the CSM to the $\alpha+\alpha+n$ three-body model. The results indicate that there is no resonant solution with a small decay width corresponding to the distinct peak observed just above the ${ }^{8} \mathrm{Be}+n$ threshold in the photodisintegration cross section of ${ }^{9} \mathrm{Be}$. However, the calculation well reproduces the recent experimental data of the energy distribution of the cross section as shown in Fig. 1 taken from Ref. [18]. In addition, by changing the attraction of the inter-three-cluster force $\left(v_{3}\right.$ is defined in Eq. (6) of Ref. [18]), the pole trajectory is calculated from a bound state to an unbound state, and the result suggests that the first excited $1 / 2^{+}$state in ${ }^{9} \mathrm{Be}$ is a ${ }^{8} \mathrm{Be}+n$ virtual state but not a resonant one.

In our recent work [23], applying a simple schematic twobody model explained above, we have investigated a virtual state in the CSM and the photodisintegration cross section. Since virtual-state poles can be solved using the Jost function method [25] explicitly, we are able to see that the virtual state has a strong influence on the photodisintegration cross sections. Seeing the scattering phase shift calculated from the continuum solutions in the CSM, we also confirmed that the virtual state is included in the continuum solutions. Using the solutions of the CSM for the continuum states in the twobody model simulating the ${ }^{8} \mathrm{Be}+n$ system, we calculated the photodisintegration cross section and obtained a similar peak with the ${ }^{9} \mathrm{Be}\left(1 / 2^{+}\right)$state at the low energy region, as shown in

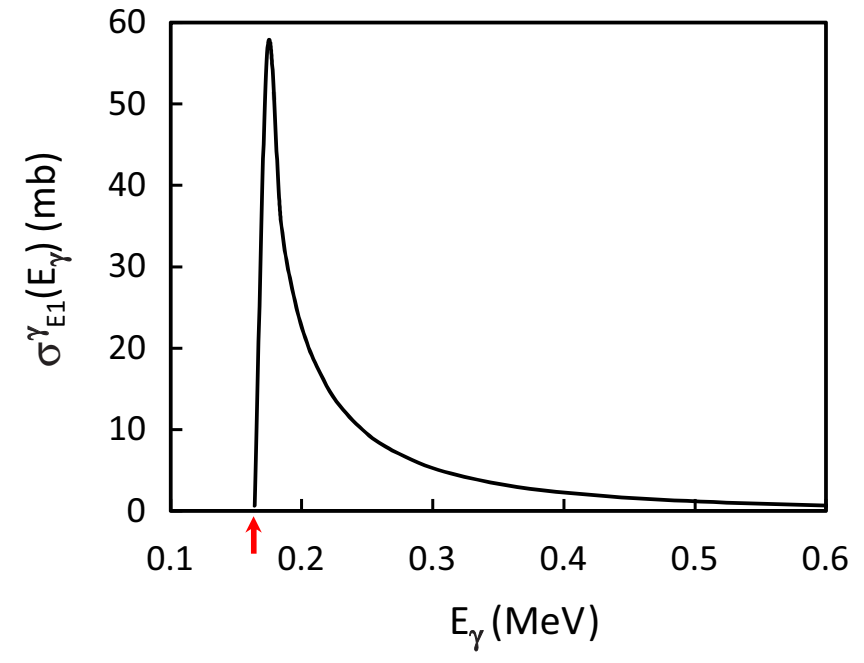

FIG. 2. The photodisintegration cross section due to the $E 1$ transition calculated with the two-body potential model with the strength $V_{1}=-1.42 \mathrm{MeV}$. The arrow indicates the threshold energy. This result is taken from Ref. [23].

Fig. 2 taken from Ref. [23]. Thus, we concluded that the twobody model describing a virtual state reproduces the shape of the photodisintegration cross section above the threshold energy similarly to the $\alpha+\alpha+n$ three-body calculation.

From Figs. 1 and 2, we see a specific energy distribution of the photodisintegration cross section. Such an asymmetric shape of the cross section is described by using the energy dependence of the resonance parameters in the $R$-matrix theory $[15,16,26,27]$. In the $R$-matrix theory, the cross section for a spin- $J$ state is approximately described with the singleresonance formula

$$
\sigma(\gamma, n)=\frac{(2 J+1) \pi}{8} \frac{\hbar^{2} c^{2}}{E_{\gamma}} \frac{\Gamma_{\gamma} \Gamma_{n}}{\left(E-\Delta-E_{R}\right)^{2}+\frac{1}{4} \Gamma^{2}},
$$

where $\Gamma_{\gamma}$ and $\Gamma_{n}$ are $\gamma$ and neutron widths, respectively. To explain the deviation from a symmetric Breit-Wigner form, the energy dependence of parameters $\Gamma_{\gamma}, \Gamma_{n}$, and $\Delta$ is introduced. Their parameters including $E_{R}$ and $\Gamma$ are chosen so as to reproduce the observed data, but it has been reported that the results are not consistent with each other [15,17].

In our calculation of the two-body model, there is no parameter except for the potential strength $V_{1}$ which determines the structure of the excited state. However, the calculated photodisintegration cross section, which includes all kinds of contributions of the final states, well explains its observed shape. Therefore, it is very interesting to investigate the origin of the peak form of the cross section.

\section{B. The origin of the peak structure}

The origin of the peak in the energy region above the threshold energy may be understood by looking into the contributions of the components of the strength function. The strength function given by Eq. (10) is decomposed into the $E 1$ matrix elements $M_{v}^{2}$ and the level density $\tilde{\rho}_{v}(E)$ of the final states. To see their energy dependences, in Fig. 3 we 

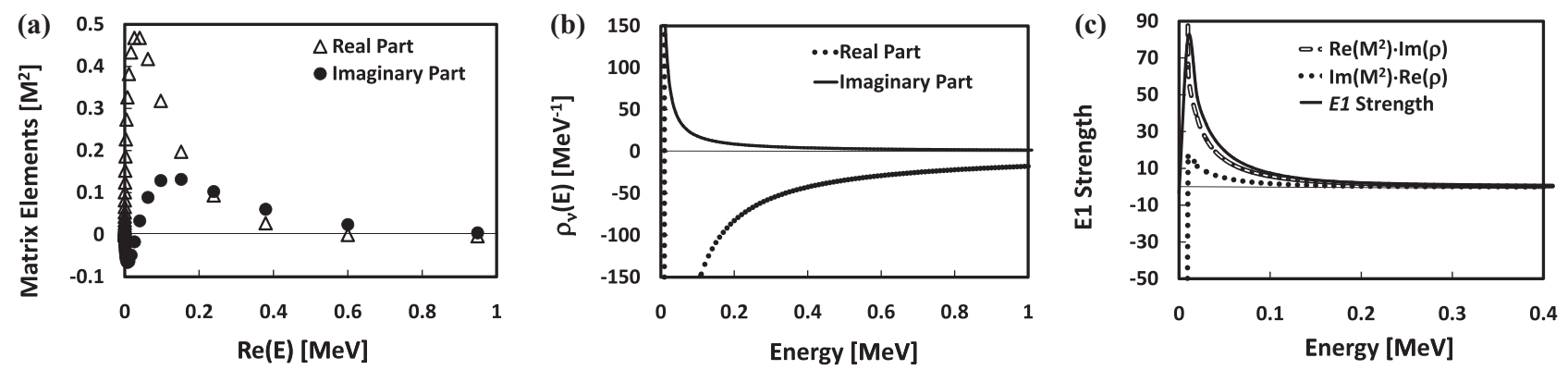

FIG. 3. Left: Matrix elements $M_{v}^{2}\left(\operatorname{Re}\left(E_{v}\right)\right)$, calculated by Eq. (11). The black and open circles represent the imaginary and real parts, respectively. Middle: Real (cross) and imaginary (black circle) parts of the level density $\rho(E)$ calculated by Eq. (12). Right: The $E 1$ strength distribution given in Eq. (10). Dotted, broken, and solid lines are $\operatorname{Re}\left(M_{v}^{2}\right) \times \operatorname{Im}(\rho(E)), \operatorname{Im}\left(M_{v}^{2}\right) \times \operatorname{Re}(\rho(E))$, and their sum, respectively.

show the matrix element $M_{v}^{2}$ of the $v$ state at the real part, $\operatorname{Re}\left(E_{v}\right)$, of the complex-scaled energy $E_{v}$ and the level density $\tilde{\rho}(E)=\sum_{\nu} \tilde{\rho}_{\nu}(E)$ at the continuous energy $E$ together with the strength function $d B\left(E 1, E_{\gamma}\right) / d E_{\gamma}$.

From the results, we can see that the real part of the matrix element $M_{v}^{2}$ and the imaginary part of $\tilde{\rho}(E)$ are dominant. They have large values at low energies, though the former goes to zero as $\operatorname{Re}\left(E_{v}\right) \rightarrow 0$. The latter shows a large concentration at zero energy. As a result, the strength function is described by $\operatorname{Re}\left(M_{v}^{2}\right) \times \operatorname{Im}(\tilde{\rho}(E))$ dominantly and shows a peak near zero energy. Thus, the peak structure of the photodisintegration cross section, shown in Fig. 2 and also by the full line in the right-hand panel of Fig. 3, can be understood from the properties of $\operatorname{Re}\left(M_{v}^{2}\right)$ and $\operatorname{Im}(\tilde{\rho}(E))$.

In our calculation, the cross section is approximately given as $\sigma \propto \sum_{v} \operatorname{Re}\left(M_{v}^{2}\right) \times \operatorname{Im}\left(\tilde{\rho}_{v}(E)\right)$, where $\operatorname{Im}\left(\tilde{\rho}_{v}(E)\right)$ is expressed as

$$
\operatorname{Im}\left(\tilde{\rho}_{\nu}(E)\right)=-\frac{1}{\pi}\left\{\frac{E_{v}^{\theta I}}{\left(E-E_{v}^{\theta R}\right)^{2}+\left(E_{\nu}^{\theta I}\right)^{2}}\right\}
$$

by using the eigenenergy $E_{v}=E_{v}^{\theta R}+i E_{v}^{\theta I}$ in the CSM and shown in the middle panel of Fig. 3. We have studied that

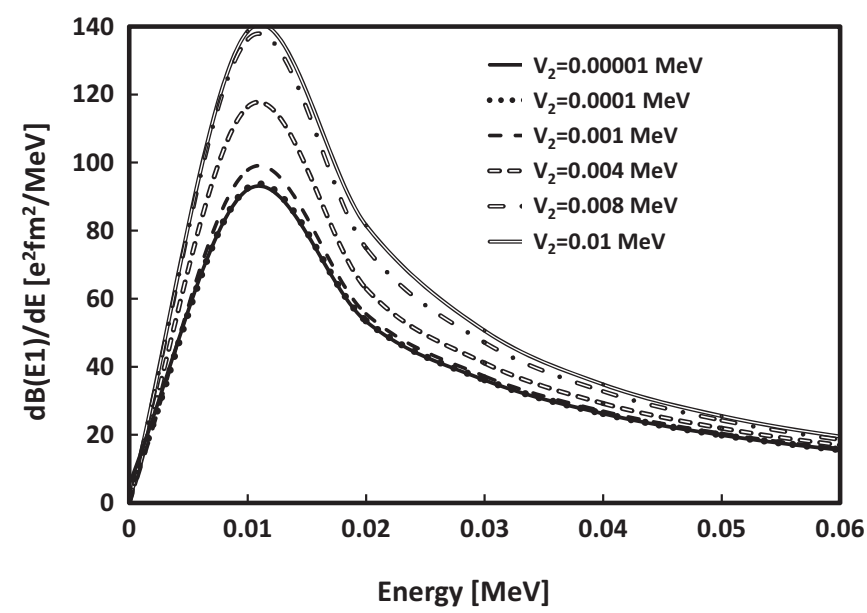

FIG. 4. The $E 1$ strength functions for the photodisintegration cross section calculated by using the wave functions of $s$ waves for different $V_{2}$ values $\left(V_{2}=0-0.01 \mathrm{MeV}\right)$ in the CSM. $\sum_{v} \operatorname{Im}\left(\tilde{\rho}_{\nu}(E)\right)$ describes the level density $\rho(E)$, which is shown to include a virtual state in the previous study [23]. It is considered that $\operatorname{Re}\left(M_{v}^{2}\right)$ and $\operatorname{Im}\left(\tilde{\rho}_{v}(E)\right)$ may correspond to numerator and denominator in Eq. (13) of the $R$-matrix calculations, respectively.

Our calculation of Eq. (10) taking sum over $v$ includes all of continuum states and also resonant states if they exist. Therefore, our calculation using the CSM describes not only a single resonance term shown in Eq. (13) but also virtualstate and many-resonance terms in addition to a background (continuum-state) term. And, the present result indicates that the asymmetric sharp peak is reproduced by the virtual state dominantly. It is interesting to investigate how the shape of the peak changes when a resonant state appears near the threshold instead of the virtual state.

\section{Difference between virtual and resonant states in shapes of the peak}

We inquire into the difference between virtual and resonant states in shapes of the peak just above the threshold energy. Because the potential of Eq. (3) reproduces no $s$-wave resonance, we add a long-range and weakly repulsive potential so as to reproduce an $s$-wave resonant state with as little influence

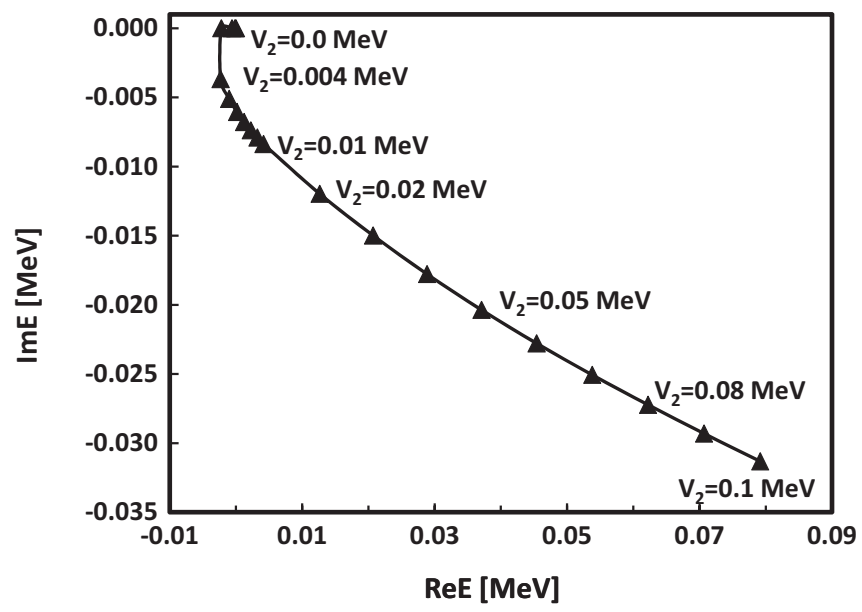

FIG. 5. Pole trajectory of the resonance state in a complex energy plane by changing $V_{2}$ potential strength. 


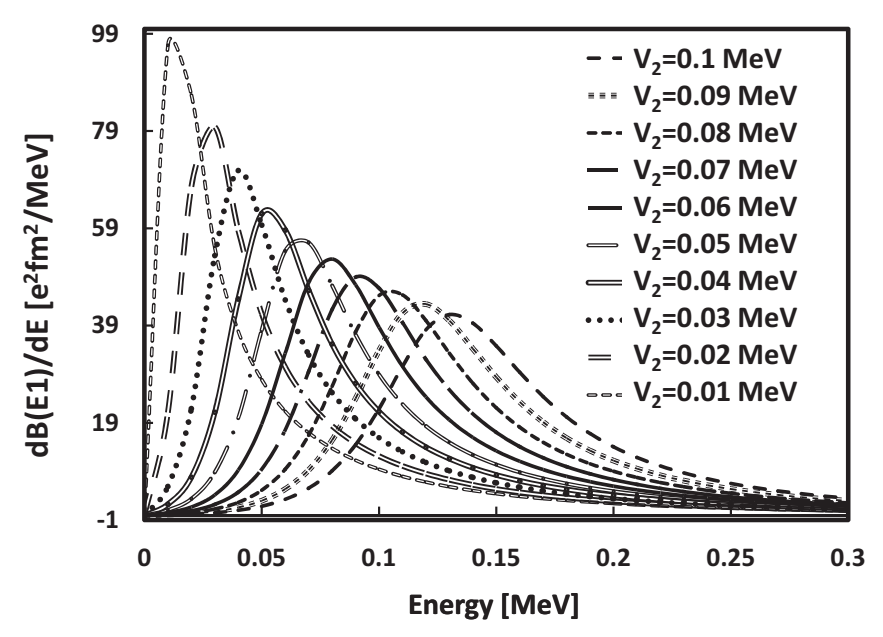

FIG. 6. The $E 1$ transition strength for different values of $V_{2}$ parameter.

on the states in the inside region as possible:

$$
V(r)=-1.42 \exp \left(-0.16 r^{2}\right)+V_{2} \exp \left(-\gamma r^{2}\right) .
$$

Parameters $V_{2} \geqslant 0.0$ and $\gamma=0.01 \mathrm{fm}^{-2}$ are taken for $s$ waves, while $V_{2}=0.0$ for the $p$-wave ground state.

Changing the potential strength $V_{2}$, we calculate the transition strength, and show the results in Fig. 4. For the range of $V_{2}=0-0.01 \mathrm{MeV}$, the $E 1$ strength functions show a similar energy distribution with a peak at almost the same energy of $0.01 \mathrm{MeV}$, though the peak height grows slightly as $V_{2}$ increases from zero. The range of $V_{2}=0-0.01 \mathrm{MeV}$ is considered to correspond to a transitional region from virtual to resonant states. To confirm this situation, we calculate the pole trajectory for $V_{2}=0-0.01 \mathrm{MeV}$ using the Jost function method [25].

As mentioned above, the Jost function method is applicable to solve not only resonant but also virtual states in two-body systems. We applied the Jost function method to calculate the $s$-wave pole trajectory for $V_{2}=0-0.1 \mathrm{MeV}$. The results are shown in Fig. 5. We see that poles for $V_{2}=0-0.03 \mathrm{MeV}$ are obtained on the negative energy axis, which mean virtual states. For $V_{2}=0.004-0.01 \mathrm{MeV}$, poles move from the real energy axis to a lower energy region of the complex energy plan. A characteristic of poles in this region, which reproduce the sharp peak of the $E 1$ strength function shown in Fig. 4, is that they are near zero energy.

When we further increase the potential strength $V_{2}$ by 0.01 $\mathrm{MeV}$ steps, the pole trajectory indicates a resonance behavior as shown in Fig. 5. At $V_{2}=0.01 \mathrm{MeV}$, the resonance energy
$[\operatorname{Re}(E)]$ is smaller than the width $[2|\operatorname{Im}(E)|]$. At $V_{2}=0.1$ $\mathrm{MeV}$, we can solve the resonance pole energy as an isolated solution in the CSM as well.

For $V_{2}=0.01-0.1 \mathrm{MeV}$, we calculate the $E 1$ transition strength and show the results in Fig. 6. It is seen that, at $V_{2}=0.01 \mathrm{MeV}$, the peak is obtained at a low energy region with a shape similar to that observed in Fig. 4. As $V_{2}$ increases, the sharp peak gradually moves from the low energy region to higher energies, and the height of the peak decreases smoothly. At $V_{2}=0.1 \mathrm{MeV}$, we see that the peak of the $E 1$ transition strength becomes a usual Breit-Wigner type which has a symmetric distribution around the resonance energy.

Coming back to the observed photodisintegration data for ${ }^{9} \mathrm{Be}$, as shown in Fig. 1, this energy distribution shows a strong asymmetric shape largely deviating from the Breit-Wigner type. Therefore, the $E 1$ transition strength shown in Fig. 6 and the pole distribution for $V_{2}>0.01 \mathrm{MeV}$ are not acceptable. This asymmetry implies the existence of a virtual state or a resonance very close to the threshold.

\section{CONCLUSION}

The photodisintegration cross section for the $1 / 2^{+}$state in ${ }^{9} \mathrm{Be}$, which was observed to have a peculiar enhancement near the ${ }^{8} \mathrm{Be}+n$ threshold energy, was discussed by using the schematic two-body potential model simulating the ${ }^{8} \mathrm{Be}+n$ system. The origin of the peak is investigated in relation to the unbound states of ${ }^{9} \mathrm{Be}$, such as virtual states, in the CSM. The contributions of the components of the strength function make clear that the real part of the matrix element and the imaginary part of the level density are dominant.

The $E 1$ strength functions for the photodisintegration cross section of the virtual state have been shown to have a peak just above the threshold energy, which is very similar to that of resonant states near zero energy. Furthermore, it is problematic that we observe such a resonance as an isolated resonant state, because the width is much larger than the resonance energy. However, if the $s$-wave neutron makes a resonance in the case of ${ }^{9} \mathrm{Be}$, it is an open problem to explain the resonance mechanism for an $s$-wave neutron to be trapped in the inside region of the nucleus.

\section{ACKNOWLEDGMENTS}

This work was supported by JSPS KAKENHI Grants No. 25400241, No. 15K05091, No. JP18K03636, and No. JP18K03660 and the National University of Mongolia's Support for High Impact Research program. This work is partly supported by the Collaborative Research Program 2018, Information Initiative Center, Hokkaido University, Sapporo, Japan.
[1] K. Ikeda, N. Takigawa, and H. Horiuchi, Prog. Theor. Phys. Suppl. E 68, 464 (1968).

[2] H. Horiuchi, K. Ikeda, and K. Katō, Prog. Theor. Phys. Suppl. 192, 1 (2012).
[3] F. Hoyle, Astrophys. J. Suppl. 1, 121 (1954).

[4] D. R. Tilley, J. H. Kelley, J. L. Godwin, D. J. Millener, J. Purcell, C. G. Sheu, and H. R. Weller, Nucl. Phys. A 745, 155 (2004) 
[5] C. W. Arnold, T. B. Clegg, C. Iliadis, H. J. Karwowski, G. C. Rich, J. R. Tompkins, and C. R. Howell, Phys. Rev. C 85, 044605 (2012).

[6] H. Utsunomiya, S. Katayama, I. Gheorghe, S. Imai, H. Yamaguchi, D. Kahl, Y. Sakaguchi, T. Shima, K. Takahisa, and S. Miyamoto, Phys. Rev. C 92, 064323 (2015).

[7] A. E. Robinson, Phys. Rev. C 94, 024613 (2016).

[8] J. Hiura and I. Shimodaya, Prog. Theor. Phys. 30, 585 (1963).

[9] P. Descouvemont, Phys. Rev. C 39, 1557 (1989).

[10] J. Casal, M. Rodriguez-Gallardo, J. M. Arias, and I. J. Thompson, Phys. Rev. C 90, 044304 (2014).

[11] V. S. Vasilevsky, K. Katō, and N. Zh. Takibayev, Phys. Rev. C 96, 034322 (2017).

[12] S. Okabe, Y. Abe, and H. Tanaka, Prog. Theor. Phys. 57, 866 (1977).

[13] W. von Oertzen, Z. Phys. A 354, 37 (1996).

[14] N. Itagaki and S. Okabe, Phys. Rev. C 61, 044306 (2000).

[15] F. C. Barker, Aust. J. Phys. 53, 247 (2000).

[16] K. Arai, P. Descouvemont, D. Baye, and W. N. Catford, Phys. Rev. C 68, 014310 (2003).
[17] V. D. Efros, P. von Neumann-Cosel, and A. Richter, Phys. Rev. C 89, 027301 (2014).

[18] M. Odsuren, Y. Kikuchi, T. Myo, M. Aikawa, and K. Katō, Phys. Rev. C 92, 014322 (2015).

[19] Y. Kikuchi, M. Odsuren, T. Myo, and K. Katō, Phys. Rev. C 93, 054605 (2016).

[20] J. Aguilar and J. M. Combes, Commun. Math. Phys. 22, 269 (1971); E. Balslev and J. M. Combes, ibid. 22, 280 (1971).

[21] S. Aoyama, T. Myo, K. Katō, and K. Ikeda, Prog. Theor. Phys. 116, 1 (2006).

[22] T. Myo, Y. Kikuchi, H. Masui, and K. Katō, Prog. Part. Nucl. Phys. 79, 1 (2014).

[23] M. Odsuren, Y. Kikuchi, T. Myo, G. Khuukhenkhuu, H. Masui, and K. Katō, Phys. Rev. C 95, 064305 (2017).

[24] E. Hiyama, Y. Kino, and M. Kamimura, Prog. Part. Nucl. Phys. 51, 223 (2003).

[25] H. Masui, S. Aoyama, T. Myo, K. Katō, and K. Ikeda, Nucl. Phys. A 673, 207 (2000).

[26] F. C. Barker and B. M. Fitzpatraick, Aust. J. Phys. 21, 415 (1968).

[27] O. Burda, P. von Neumann-Cosel, A. Richter, C. Forssén, and B. A. Brown, Phys. Rev. C 82, 015808 (2010). 